\title{
Caring Newborn on Ventilator - Concepts, Comprehensions and Core Competencies
}

\author{
Vaidyanathan Radha ${ }^{1}$, Pratibha A Chandekar ${ }^{2}$ \\ I'Professor, Child Health Nursing, Maharashtra University of Health Sciences, Nashik \& Paediatric Matron, \\ Command Hospital, Pune, Maharashtra, India) \\ 2 (Dean, Allied Health Sciences, Principal, CON, Pravara Institute of Medical Sciences, Loni, Ahmednagar, \\ India)
}

\section{Introduction}

We seem to live in an age of limitless technological marvels in neonatal ventilation. It will not be out of place to say that the exponential growth in this sector is crafted in the dark by the skilled hands and minds using Trial and Error Principle of Science since 1971, when the first CPAP was introduced by Dr. Gregori, an anesthesiologist.

The first recorded reference to assisted ventilation is in the second Book of Kings 4:32-35, "And when Elisha was come into the house, behold the child was dead ... and he went up and lay upon the child and put his mouth upon his mouth, and his eyes upon his eyes, and his hands upon his hands: and he stretched himself upon the child; and the flesh of the child waxed warm." ${ }^{[1]}$ Dr. Virginia Apgar highlighted, "They didn't record that he blew, but he did!" was a significant medical record.

Today we have an extensive and commanding exposition over the machine but not yet on the marvelous baby under the machine. This paper aims to simplify the newborn chest physiology, principles, monitoring and management strategies for caring ventilated newborns.

\section{General Principles}

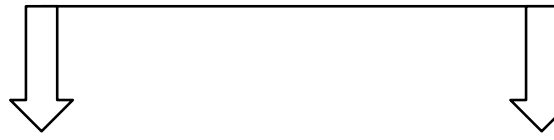

Process of Ventilation
Newborn

Dimension

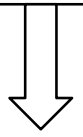

Machine

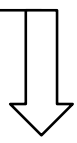

Manpower

\section{(2.1) Ventilation}

* It is the process of gas exchange to move oxygen from the air to the blood and $\mathrm{CO} 2$ from the blood to the air is known as ventilation.

* It is the product of breathing frequency (f) and tidal volume (VT).

- It refers primarily to the amount of carbon dioxide exchanging at the alveolar level.

* It is a tool to tide over the time till the lung heals and become functional.

* There are two goals of ventilation: Appropriate Oxygenation \& Ventilation

- Oxygenation is affected by several factors such as the inspired oxygen concentration $\left(\mathrm{FiO}_{2}\right)$, mean airway pressure (MAP), the area of and diffusion across the gas exchange surface)

\section{(2.2) Newborn dimension}

* Newborn chest wall is very compliant: This implies that the external intercostals muscles serve to stabilize the chest wall.

* When respiratory distress develops, pulmonary compliance is reduced.

- Contraction of the diaphragm in the presence of lung disease will result in a decrease in intra thoracic pressure, intercostals and sternal retractions rather than inflation of the lungs.

* The diaphragm inserts more horizontally in infants than adults contributing to the development of lower rib retractions particularly when the newborn is supine. The greater the retractions present, the more the diaphragm will need to contract to generate an adequate tidal volume, making ventilation inefficient and it will be necessary for the diaphragm to shorten and move as much as $130 \%$ of normal to generate the required tidal volume.

* Difference in lung mechanics during inspiration and expiration for a newborn with a lung disease, where the chest wall moves outward during expiration and inward for inspiration that is not the case in normal newborn lungs. 
* Neonatal Lung Fragility: Chest wall instability, fewer alveoli and impaired production of pulmonary surfactant still poses additional challenges

\section{(2.3) Mechanical Ventilator}

* Mechanical Ventilation is used to replace the function of the diaphragm and thoracic chest wall muscles.

- Concept of AYRES T - piece principle: If you completely occlude one end, you produce inspiration by diverting gas flow to the baby. If the occlusion is released completely, gas flow through a path of least resistance and produces expiration.

- Machine is user friendly with no gizmo nightmares of a video game and affordable.

* A comprehensive NICUBATOR is ideal with all compact accessories.

\section{(2.4) Manpower}

* Nurse: Newborn ratio must be $1: 1$ or to a maximum of $1: 2$.

* Nurse having knowledge and skill with a positive ATTITUDE

\section{Principles of caring for a Ventilated Newborn}

Irrespective of the technique or mode of ventilation chosen, the nursing principles are to identify the most appropriate device, technique, and strategy to:

(3.1) Achieve and maintain adequate pulmonary gas exchange

(3.2) Minimize the risk of lung injury / Recognition and Prevention of VALI (Ventilator

Associated Lung Injury)

(3.3) Reduce newborn Work Of Breathing (WOB) and

(3.4) Optimize newborn comfort

- Appropriate oxygenation<smiles>C1CCC(C2CCCC2)C1</smiles>

- Appropriate ventilation

\subsection{Ventilator and its accessories}

\section{Equipments required for babies on ventilator}

\subsection{Nursing Care related equipments}

(a) Clinical Monitoring devices: Thermometers, Weighing scale, Pulse Oximeter, NIBP, TCB ?, Oxygen analyzer, Glucometer, TCPO2 and TCPCO2, Intra arterial BP monitor

(b) Temperature control devices: Warmidifier (Humidification and warming), Incubators, Radiant Warmers

(c) Ressuscitation devises: Ressuscitation bag, Laryngoscope

(d) Therapy related devices: Suctioning apparatus complete, Infusion Pumps, Phototherapy units,

Continuous supply of Oxygen and Compressed Air

(e) ADL care items: Feeding, Cleaning, Physiotherapy, Miscellaneous

(f) Disinfection articles

\subsection{Infrastructure of the Unit}




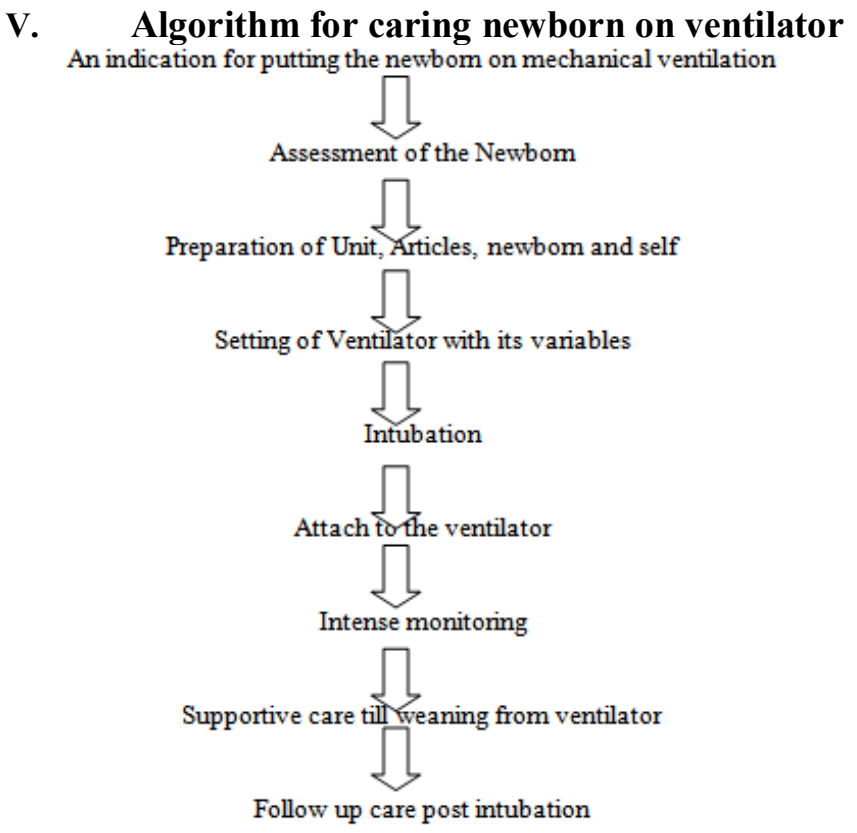

\section{Assessment of Newborn (Monitoring)}

It is the single most effective tool for efficient ventilation especially in the first few hours. A skilled nurse's "feel good or bad factor" on newborn's clinical picture is to be taken as the "Eleventh Commandment" for deciding management strategies for the day.

- Provides early evidence of potentially dangerous conditions - gas trapping and hyperinflation

- Helps to determine optimal PEEP

- Gives immediate feedback on the effects of changes in ventilator parameters

- Real-time monitoring should decrease the need for and frequency of many ancillary tests, such as chest radiography and blood gas analysis, thus decreasing the cost of health care.

- Allows better control of some variables

\subsection{When to Monitor?}

1. Before you touch the Ventilator and the Baby

2. Once the newborn is ventilated and then on...

6.1. Before you Touch the Ventilator.....

(a) Look at the blood gas result.
(b) Look at the baby.

- Is the chest moving?

- What's the air-entry like?

- Is the baby very tachypnoeic or is the baby apnoeic?

(c) Look at the ventilator.

- What tidal volume (VT) is the baby getting?

- Is there a significant leak?

- Is it set up properly with an appropriate inspiratory time and with appropriate pressures?

(d) Look at the nursing flow chart.

- How stable has the baby been over the past few hours or days?

- Are there lots of secretions?

\subsection{On Ventilation and thereon monitoring}

\section{Ideal Monitoring Features}

6.2.1. Proximal Airway Monitoring

6.2.2 Real-time Pulmonary Graphics 


\subsubsection{Proximal Airway Monitoring}

Clinical Methods are:

* Physical Examination

* Vital Signs update

* ABG Analysis (Hematological / Bio chemical), Blood sugar \& Calcium

* Chest Radiograph interpretation

- done at start of ventilation, after surfactant, after each tube change

- 8 Ps - Position of baby, Parenchyma, Pleura, Phrenic angles, Perihilar area, Pericardium, Peripheries, Plastics (ET tubes, umbilical catheter)

* Measurement of pulmonary function variables (Biochemical)

* Assessment of ventilation: Oxygenation and airway patency

* Bacteriological surveillance : Blood culture, ET culture, Suction catheter tip

\subsubsection{Real-time Pulmonary Graphics}

Real-time Pulmonary Graphics:

* Waveforms

* Loops

* Mechanics

* Trending

6.3 Aspects of Monitoring / Observation

(i) Continuous monitoring techniques:

- Pulse Oximetry

- Transcutaneous Gases

- Pulmonary Mechanics

(ii) Intensive monitoring

- Vitals (look for PDA especially in preterm), FiO2, Weight, Abd girth, blood sugar, PCV

- Strict Intake - output

(iii) Anticipate problems and closely observe

- Activity, behavior, signs of sepsis, apnea

(iv) Hemodynamic Stability Color, Saturation, CRT, CVP, urine output

(iv) Non adjunctive monitoring:

* Sophisticated devices

- Transcutaneous Monitoring Devices Clark Electrode (measure oxygen tension)

- Stowe Severinghaus CO2 electrode (Partial pressure of CO2)

* End Tidal $\mathrm{CO} 2$ monitoring

* Airway Pressure monitoring

$\circ \quad$ MAP, Transpulmonary Pressure (TPP)

\section{(7.1) Positioning}

\section{Supportive care of Ventilated Newborn}

* Place healthy lung on the dependant position as this result in increased perfusion of the good lung segments and possible improvement of the involved lung.

* Change position: Supine, right or left lateral every $2-4$ hourly and prone if feasible

- Encourages postural drainage when indicated

* Passive physiotherapy - Plastic cup with padded rim or soft circular mask are used to apply kinetic energy to chest wall by rhythmically striking the thorax over the lung segment.

* Augment with nebulisation with or without aerosolized medicines

\section{(7.2) Prevention of nosocomial infection}

\section{(7.3) Surveillance of flora}

- Routine :Weekly swabs from various sites of NICU

- Specific patient-related

- Endotracheal tube tips

- Intravenous long-line tips

- Strict aseptic procedures

- Carbolysation / disinfection of all equipments / items entering NICU 


\section{(7.4) Provision of in-utero like milieu}

○ Nesting, Gentle handling

- Reduce ambient noise levels

- Control NICU environment - Optimum Lighting, < 45 DB

○ Thermal Comfort : Thermoneutral environment

\section{(7.5) Minimizing Oxygen demand}

* Treat fever, pain and agitation x SOS as they increase oxygen consumption

* Neutro Thermal Environment

* Pharmacologic paralysis

* Attend to crying, excessive movements of limbs, discomfort through linen, cotton, gauze, chill hands, noise, vibrations from bowls, articles of care, etc increase BMR and hence oxygen demand.

* Schedule care activities at one go - club together

* Approach softly and gently

\section{(7.6) Humidification and Pulmonary Hygiene}

ET Intubation bypasses the natural gas humidification at the upper airway level. Inadequate humidification of the inspired air may result in increased volume and viscosity of secretions and this increases risk of airway obstruction.

\section{(7.7) Suctioning}

* Strictly a sterile procedure

* Duration is short - entire procedure not more than $10-20 \mathrm{sec}$

* Catheter should not occlude more than $2 / 3^{\text {rd }}$ diameter of ET Tube

\section{(7.8) ABG Monitoring}

\section{(7.9) Fluid and Nutritional support}

Positive Pressure Ventilation is associated with increased levels of circulating ADH, so the kidneys will retain free water. Hence the newborn with compromised ventilation should receive about 66 to $75 \%$ of calculated maintenance fluid requirements unless presented with significant dehydration or hypovolemia. Generous fluid administration may contribute to the development of pulmonary edema and worsening of respiratory failure.

\section{(7.10) Sedation, Analgesia and Paralysis}

* Bolus dose with infusion is more effective to achieve therapeutic drug levels

* Rule out the cause of agitation before analgesia like ET tube displacement, hypoxia

- Short acting Benzodiazepines

Midazolam $0.1-0.2 \mathrm{mg} / \mathrm{kg}$ IV

Lorazepam $0.05-0.1 \mathrm{mg} / \mathrm{kg}$ is long acting

- Fentanyl and Morphine $(0.1 \mathrm{mg}-0.5 \mathrm{mg} / \mathrm{kg}$ continuous infusion $)+$ Muscle relaxant

- Ketamine for dissociative anesthesia

- Pancuronium $(0.1 \mathrm{mg} / \mathrm{kg})$, Vencuronium $(0.1 \mathrm{mg} / \mathrm{kg} / \mathrm{hr})$ - for muscle relaxation

- Paralysis necessary if high peak inspiratory pressure is created

\section{(7.11) Weaning}

\section{(i) Evaluation for ventilatory weaning}

* Stable infant for at least $24 \mathrm{hrs}$ with no inotropic support

+ $\mathrm{FiO} 2$ reduced to $50 \%$ with $\mathrm{PaO} 2$ value remaining $50-80 \mathrm{mmHg}$.

* Oxygenation indices stable $-\mathrm{ABG}, \mathrm{CXR}$

* Spontaneous breathes should increase in number and spontaneous breath tidal volumes should equal mechanical breath volumes $(5-7 \mathrm{ml} / \mathrm{Kg})$. The decreasing mechanical rate should be matched by infants increasing rate.

* Monitoring of TV should have shown a progressive decrease of rate and pressure must be accompanied by a sustained $\mathrm{PaCO} 2$ of $40-50 \mathrm{~mm} \mathrm{Hg}$

(ii) Weaning Procedure

* Rate settings should be decreased hourly (newborns tolerate rate of wean when they do not require more $\mathrm{FiO} 2)$ 
* When the ventilator rate is less than $10 \mathrm{bpm}$ the infant should be extubated

* Weaning vary from newborn to newborn from days to week or more

* Gradual

* Keep close watch for complications

* If repeated intubations are required, dexamethasone $0.5 \mathrm{mg} / \mathrm{Kg} /$ day BD 12- $24 \mathrm{hrs}$ before planning extubation to reduce airway edema

* Aminophylline may be required if wt $<1500$ gm

* Oral Feeding withheld atleast 4 hrs before extubation to avoid aspiration of stomach contents

(7.12) Site and type of IV access

(7.13) Documentation

(7.14) Follow up care

- $\quad$ Put the baby on headbox with same or $5 \%$ more FiO2 - about $8 \mathrm{~L} / \mathrm{min}$

- $\quad$ Repeat ABG after $1 / 2-2$ hrs

- $\quad$ Take - CXR

- Chest Physiotherapy

- Feeding resumed after $8-12$ hrs after the infant can approximate the vocal cord as evidenced by audible crying

\section{(7.15) Emotional Support \\ (7.16) Good Communications}

\section{Conclusion}

Neonatal Ventilation is more helpful if started well in time rather than a last desperate measure. Nurse must be sensitive to the newborns micro responses and support all its developmental needs. It is the proficiency in interpreting the various communication of the child through the monitors, ventilators and significant changes as and when they occur will attain the purpose of ventilating a child. In other words, Caring Infants on Ventilation should endeavor us for: The ultimate method for safe and effective artificial ventilation of the newborn lung, especially when it is diseased or anatomically extremely immature, has yet to be devised.

\section{References}

[1]. Mark C Mammel MD’ New Modes of Neonatal Ventilation: Let There be Light Journal of Perinatology (2005) 25, 624-625.

[2]. Sankar MJ, Sankar J, Agarwal R, Paul VK, Deorari AK. Protocol for administering continuous positive airway pressure in neonates. Indian J Pediatr 2008; 75:471-478.

[3]. Sinha SK \& Donn SM. Advances in neonatal conventional ventilation. Arch Dis Child Fetal Neonatal Ed $2000 ; 75$ : F135-F140.

[4]. Abubakar K \& Keszler M. Effect of volume guarantee combined with assist/control vs. synchronized intermittent mandatory ventilation. J Perinatol 2005;

[5]. The HIFI study group. High-frequency oscillatory ventilation compared with conventional mechanical ventilation in the treatment of respiratory failure in preterm infants. $N$ Engl J Med 1989; 320: 88. 